\title{
TypOn: the microbial typing ontology
}

\author{
Cátia Vaz ${ }^{1,2^{*}}$, Alexandre P Francisco ${ }^{1,3}$, Mickael Silva $^{4}$, Keith A Jolley ${ }^{5}$, James E Bray ${ }^{5}$, Hannes Pouseele ${ }^{6}$, \\ Joerg Rothganger ${ }^{7}$, Mário Ramirez ${ }^{4}$ and João A Carriço ${ }^{4}$
}

\begin{abstract}
Bacterial identification and characterization at subspecies level is commonly known as Microbial Typing. Currently, these methodologies are fundamental tools in Clinical Microbiology and bacterial population genetics studies to track outbreaks and to study the dissemination and evolution of virulence or pathogenicity factors and antimicrobial resistance. Due to advances in DNA sequencing technology, these methods have evolved to become focused on sequence-based methodologies. The need to have a common understanding of the concepts described and the ability to share results within the community at a global level are increasingly important requisites for the continued development of portable and accurate sequence-based typing methods, especially with the recent introduction of Next Generation Sequencing (NGS) technologies. In this paper, we present an ontology designed for the sequence-based microbial typing field, capable of describing any of the sequence-based typing methodologies currently in use and being developed, including novel NGS based methods. This is a fundamental step to accurately describe, analyze, curate, and manage information for microbial typing based on sequence based typing methods.
\end{abstract}

Keywords: Ontology, Knowledge representation, Microbial typing methods

\section{Introduction}

It is widely known that different strains from a given bacterial species may have distinct phenotypic behaviors, such as a higher capacity to cause invasive disease, to asymptomatically colonize the host or to present resistance to antimicrobials [1]. Such distinguishing characteristics can be usually attributed to lineages identified at the level of the genotype. Microbial typing refers to the methodologies used to identify these lineages and define them at sub-species level. Microbial typing has important implications in several health related fields such as surveillance of infectious diseases, outbreak investigation and control, identification of pathogen reservoirs, and studies on pathogenesis [2]. Traditionally these methodologies were based on characterizing a limited number of markers. These markers can be phenotypic characteristics, such as the presence of certain structures on the bacterial surface [3], or genetic characteristics, such as the presence on the bacterial genome of DNA sequences

*Correspondence: cvaz@cc.isel.ipl.pt

1 INESC-ID, R. Alves Redol 9, 1000-029 Lisboa, Portugal

2 Instituto Superior de Engenharia de Lisboa, Instituto Politécnico de Lisboa, R.

Cons. Emídio Navarro 1, 1959-007 Lisboa, Portugal

Full list of author information is available at the end of the article that are recognized and cleaved by specific enzymes, generating band patterns by gel electrophoresis [4]. More recently, due to the low cost and increasing availability of DNA sequencing technologies, the development of typing methods became focused on the use of DNA sequence information.

Although these methods revolutionized microbial typing, through the creation of novel, unambiguous and easily understandable nomenclatures for human use, the existing databases still lack interfaces for machinereadable formats, which can be used for automated data submission and querying. An ontology describing the concepts and relationships for sequence-based typing methods can thus provide a powerful tool in the field. Sharing data annotated in a common language, and in a semantically rich machine-readable format, will allow a better integration of the data existing in databases of sequence-based typing methods, epidemiological information and the novel NGS data being produced [5]. Furthermore, it can facilitate comparison of different typing schemas, and allow users to mine, in an effective way, the ever-growing public data.

In this paper, we describe the design of TypOn, a microbial typing ontology. TypOn was developed from a 
previous prototype ontology [6], and focuses on sequencebased typing methods, including novel NGS methodologies. We discuss the connection of TypOn to existing ontologies, how to use it to annotate data already publicly available, and the methods to effectively query it.

\section{Domain description}

Several typing methods have been used in outbreak detection and epidemiological surveillance ranging from phenotypic methods to fragment based methods and sequence based methods [5,7].

Multilocus Sequence Typing (MLST) [8,9] is a widely adopted methodology to type several diferent species of microorganisms. This method is based on determining the sequence of internal fragments of multiple (typically seven) loci encoding proteins with housekeeping functions. By a locus we understand a specific location in the chromosome, where different sequences occupying a given locus define distinct alleles of that locus. Each set of loci defining an MLST scheme is proposed by a group of researchers that usually also provide evidence supporting its discriminatory power and suitability for its intended purpose. When applying the methodology, the set of alleles identified at the loci considered define a sequence type (ST), a key identifier with this methodology. The loci chosen are usually different for each species, although some species may share some or even all loci in their MLST schemas. The number of loci can be also variable and can be greater or smaller than the seven loci more commonly adopted. MLST's large appeal for the community was the reproducibility and portability of results, which allowed the deployment of databases for several bacterial species [10-12]. The strain nomenclature developed by MLST facilitated the global tracking and immediate comparison of microbial strains in clinical and research settings.

Another sequence-based method that derived its success from a common nomenclature and the ease of strain classification, was spa typing for Staphylococcus aureus [13], an important pathogen that is one of the major causes of nosocomial infections [14]. This method is based on repeat sequences present at a single locus, the spa gene. These repeats are short sequences of DNA (in the case of spa about thirty nucleotides) that, although sharing consensus characteristics, can be variable in their sequence. Their number is also variable and, in the case of spa typing, this is expressed by a string of numbers representing the identity and number of repeats present at each spa allele. An updated list of identified repeats and spa types can be found at http://spaserver.ridom.de/.

Multilocus Variable Number of Tandem Repeats Analysis (MLVA) [15] is a method that is based on the number of repeat patterns present on several defined locus that are, similarly to MLST, defined in a schema. Several schemas are also available in multiple websites such as http://www.mlva.eu/, http://www.mlva.net/ or http:// www.miru-vntrplus.org/.

With the advent of Next Generation Sequencing (NGS) technologies, and the ability to produce a draft genome sequence of a microbial strain in a couple of days instead of weeks or months, researchers can use this information to classify the strain according to new or previously available sequence-based typing methods. Furthermore, novel typing methods are being developed that are able to probe tens, hundreds or even thousands of different loci across the genome [16], effectively extending the MLST concepts. An example of such a method is ribosomal MLST [17]. Other whole genome methodologies probe the genome for Single Nucleotide Polymorphisms (SNPs) when compared to reference genomes $[18,19]$ in order to discriminate strains.

The main goal of typing methods is the characterization of individuals existing in a given sample. The sample under study can be recovered from sick or healthy subjects or directly from the environment. The first process is the isolation of the microorganisms to be characterized from the sample collected. Each individual, or in the case of bacteria, each colony isolated from the microbial population then becomes an "isolate", referring to the process of isolating it from a sample that contains many microorganisms potentially representing distinct species. In particular each isolate can then be identified at subspecies, species, or genus level, identifying it as belonging to a given taxonomic unit, i.e., taxon. Moreover, since most microorganisms reproduce asexually the subsequent propagation of this isolate in the laboratory as an axenic culture, would be expected to generate a clonal population.

Each isolate can be associated with typing information and ancillary details. The isolate can have the nucleotide sequence of its genetic material determined and multiple typing characteristics defined through different typing methods, such as MLST, spa typing or MLVA [15]. There are several categories of typing methods. For instance, although MLST and spa typing are both genotypic methods, MLST is a multi-locus typing method while spa typing is a single-locus typing method.

For the specific case of multi-locus typing methods, several schemas have been defined as indicated above. Each schema is characterized by the (possibly ordered) set of loci selected for a given taxon, usually defined at the species level. Each schema is then administrated by one or a group of microbiologist, or by an institution, and each isolate's ancillary data and respective typing information are deposited in public repositories and validated by a curator. Different schemas can be defined for the same taxon and an ontology will facilitate understanding the relationships between different schemas. Ancillary data 
include information about the place where the microorganism was isolated, information about environment or host, and other possible contextual details. Later on, we will discuss how this information can be added and annotated in the context of TypOn.

We also note that data ownership is a particular delicate issue in the surveillance of communicable diseases and, as we will discuss later, the approach proposed in this paper allows a straightforward implementation of the agreed upon policies. The sensitive nature of the information and the ethical issues associated can be also safeguarded through the application of suitable access control levels based on the ownership structure embedded in the ontology.

\section{Microbial typing ontology}

TypOn is an OWL ontology for describing microbial typing, focusing on sequence based methods. Such a description will provide a universal framework for the exchange of information on the microbial typing field, allowing the integration of data coming from the numerous and disparate online databases.

In the next Section, we describe and discuss the TypOn ontology and its suitability for describing knowledge in the microbiology typing methods domain. Later, we illustrate how to annotate and query microbial typing data and information using TypOn.

\section{Ontology modeling}

The main concepts and properties defined in TypOn are depicted in Figures 1 and 2. The ontology, which is an extended version of a previous prototype ontology [6], is available at http://purl.phyloviz.net/ontology/ typon. In this current version, we added new concepts and refined existing ones, based on comments made by domain experts, including microbiologists and industry partners. The aim of TypOn is to represent knowledge about any of the currently used sequencebased microbial typing methods. TypOn can be reused as well as expanded, whenever new requirements and new technologies demand it. For backwards compatibility, older versions can still be used by explicitly stating the TypOn version. For instance, version 20140606 may be accessed and referenced as http://purl.phyloviz. net/ontology/20140606/typon. This ontology has been developed in the context of the Patho-NGen-Trace project http://patho-ngen-trace.eu/project/ as a way to standardize microbial data exchange between online databases and current software using those databases.

Ongoing developments, new versions, as well as use cases and examples, can be found in the project repository https://bitbucket.org/phyloviz/typon, and documented in the project wiki https://bitbucket.org/phyloviz/typon/ wiki. The base URL for TypOn, http://purl.phyloviz.net/ ontology/typon, redirects transparently to the last stable version of the ontology, in RDF/XML format, located in the master branch in the development repository.

The current version of TypOn has 44 classes, including those imported from other ontologies, and 47 properties, as shown in Table 1.

Although we will discuss examples of bacterial typing, we believe that TypOn is equally applicable to typing methods used to characterize any microorganism. Naturally, this implies that other loci, in addition to those used in the examples, will have to be entered into the ontology and new schemas will have to be defined. The ontology offers a flexible framework with which the existing and future sequence typing methods can be described and the examples are meant to illustrate its application as well as its flexibility. As described above, the first process in

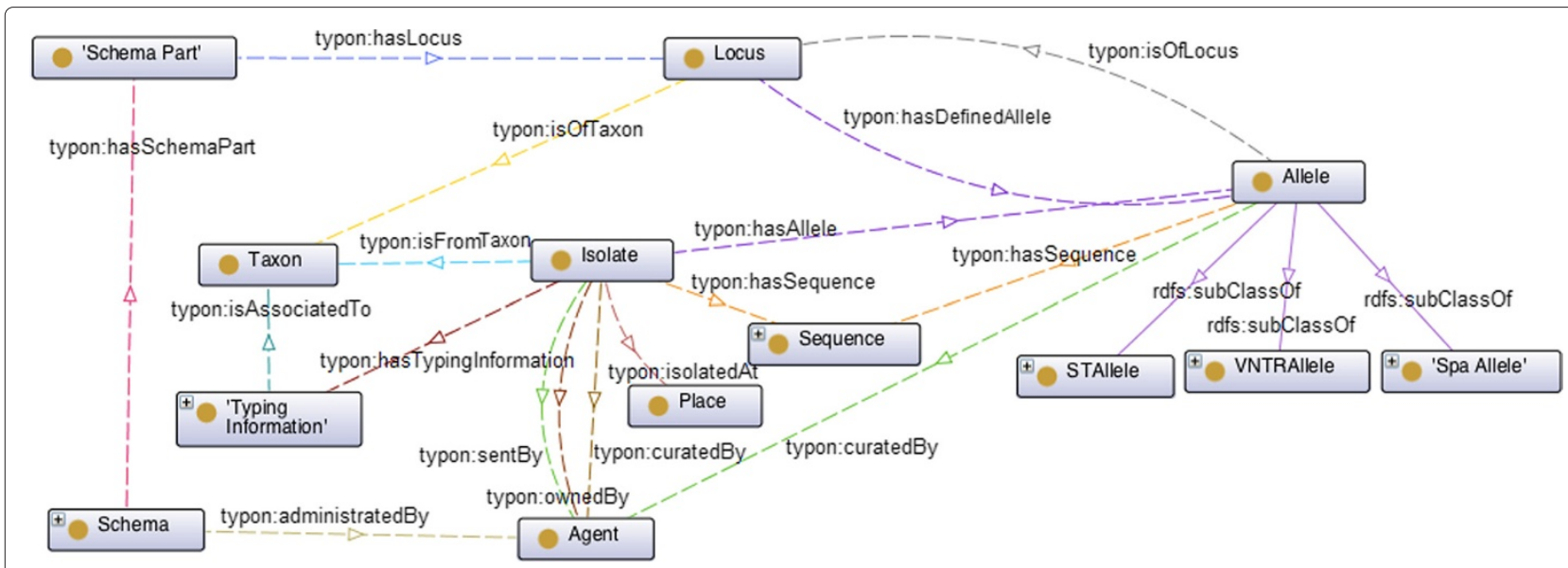

Figure 1 The Isolate concept and its object properties. Dashed lines represent object properties and solid lines represent subclass relations, e.g., STAllele is-a Allele. Properties hasLocus and isOfLocus have cardinality of exactly one. All other properties do not have any cardinality restriction. 


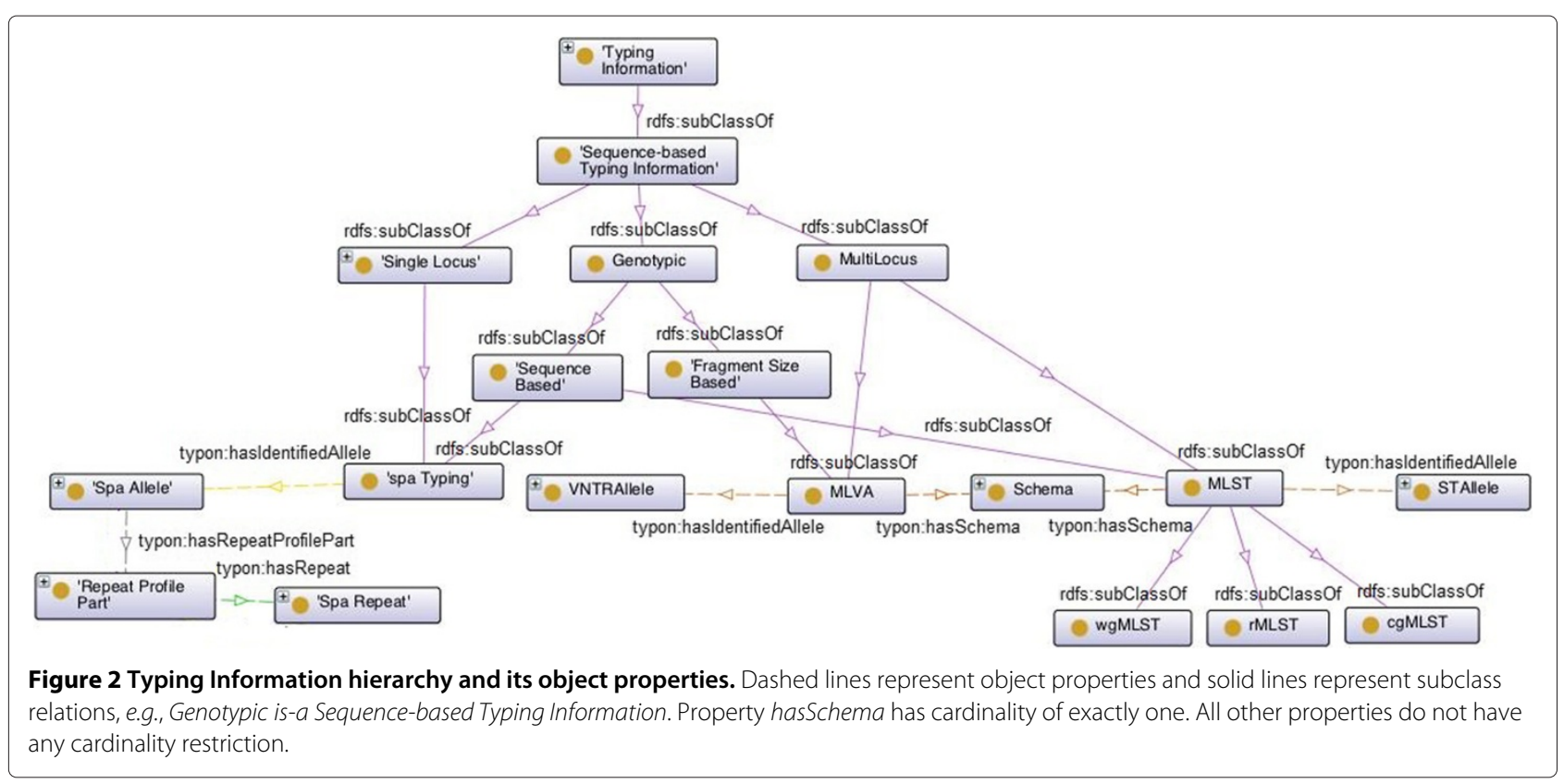

microbial typing is the recovery of the microorganisms to be characterized from the sample collected and, thus, Isolate is a main concept in TypOn and it is characterized by several properties.

Figure 1 shows an overview of the Isolate class and its related concepts and properties.

Each Isolate when identified at subspecies, species, or genus level, belongs to a certain Taxon, a relation that we express through the property isFromTaxon. The Taxon

\section{Table 1 Statistics concerning concepts and properties either defined in TypOn or reused from other ontologies/vocabularies}

\begin{tabular}{|c|c|c|c|}
\hline Ontology & Concepts & $\begin{array}{c}\text { Object } \\
\text { properties }\end{array}$ & $\begin{array}{c}\text { Data } \\
\text { properties }\end{array}$ \\
\hline $\begin{array}{l}\text { Microbial Typing } \\
\text { Ontology (TypOn) }\end{array}$ & 24 & 21 & 25 \\
\hline $\begin{array}{l}\text { Basic Formal } \\
\text { Ontology (BFO) [20] }\end{array}$ & 8 & 1 & 0 \\
\hline $\begin{array}{l}\text { Sequence Ontology } \\
\text { (SO) [21] }\end{array}$ & 6 & 0 & 0 \\
\hline $\begin{array}{l}\text { Environment Ontology } \\
(\text { EnvO) [22] }\end{array}$ & 2 & 0 & 0 \\
\hline $\begin{array}{l}\text { Ontology for Biomedical } \\
\text { Investigations (OBI) [23] }\end{array}$ & 1 & 0 & 0 \\
\hline $\begin{array}{l}\text { Uniprot Core } \\
\text { Ontology (UNIPROT) [24] }\end{array}$ & 1 & 0 & 0 \\
\hline $\begin{array}{l}\text { Friend of a } \\
\text { Friend (FOAF) [25] }\end{array}$ & 1 & 0 & 0 \\
\hline $\begin{array}{l}\text { The DBpedia } \\
\text { Ontology [26] }\end{array}$ & 1 & 0 & 0 \\
\hline
\end{tabular}

concept is reused from the Uniprot Core Ontology [27] for classifying life forms. Moreover, we define that each Isolate is an Organism, a concept that is reused from the Ontology for Biomedical Investigations (OBI) [28]. We also know the Place from where each Isolate was recovered, which we describe through the property isolatedAt. As with the Taxon concept, the Place concept is reused from another ontology, in this case the DBpedia ontology [29]. One can also define the environment material or system where a given Isolate was collected. These concepts are already found in the environment ontology (EnvO) [22] and, hence, we reused them and we add the properties isolatedOnMaterial and isolatedOnSystem, relating these concepts with the concept Isolate. Each Isolate, can have Sequence information, i.e. the nucleotide sequence of its genetic material, and Typing Information. The property hasTypingInformation commonly has cardinality higher than one for each Isolate, since several typing methods, such as MLST or MLVA, can be applied to the same Isolate. Later we will present an example of an isolate with both MLST and spa typing information.

In this context, it is important to note that TypingInformation is the root of a class hierarchy (see Figure 2). This hierarchy can be extended by including new, and already known, typing techniques, such as phenotypic information related to antibiotic susceptibility. In particular, we are able to distinguish different categories of typing methods. Let us consider MLST and spa Typing. Both are Genotypic methods, but the first is a Multilocus method while the latter is a Single Locus method.

Let us consider the concepts Locus, Allele, Schema and $M L S T$, as depicted in Figure 3. In MLST we can have 


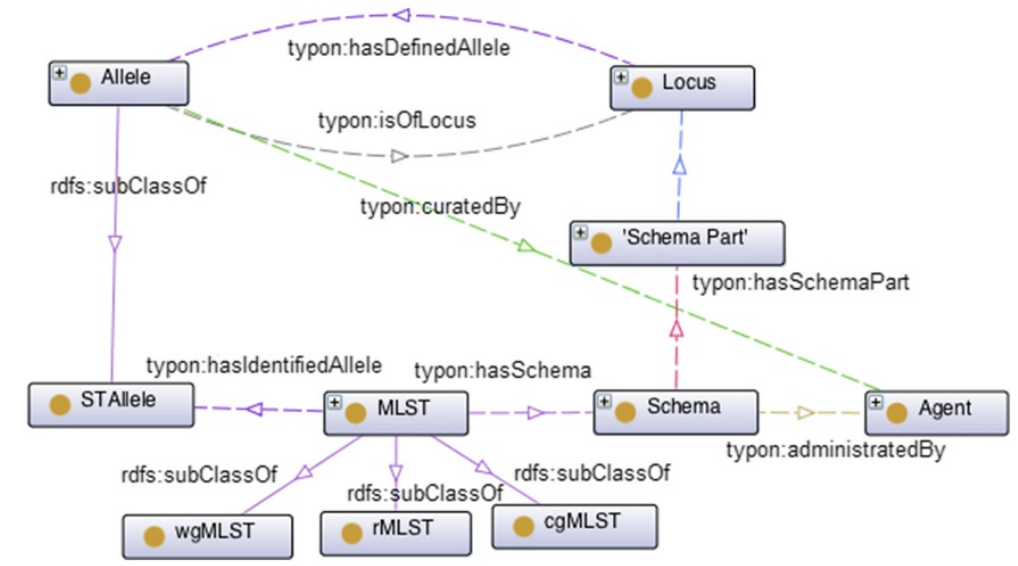

Figure 3 MLST typing information. Dashed lines represent object properties and solid lines represent subclass relations. Property is OfLocus has cardinality of exactly one. All other properties do not have any cardinality restriction.

several typing schemas administrated by some Agent, a concept reused from FOAF ontology [25], for instance the database curator, and composed by a set of Schema Parts. Such schemas are represented through the class Schema, which has associated the property hasSchemaPart. The Schema Part concept allows us to identify a particular Locus and provide the index order for that locus in the underlying schema through object property hasLocus and data property index, respectively. In the case of $M L S T$, each locus identifies a region within the coding sequence of an housekeeping gene. Thus, the object property hasLocus associated to the Schema Part concept has cardinality of exactly one.

As depicted in Figure 1 and indicated above, each Isolate may have been characterized by more than one typing method. In the case of MLST, this kind of typing information can be subject to different schemas, resulting in different sequence types, which are characterized by the alleles found at each locus. Therefore, in our ontology, we associate to each MLST both a schema and a set of observed alleles through properties hasSchema and hasIdentifiedAllele, respectively. The property hasSchema has cardinality of exactly one and, hence, MLST and MLVA instances must be related with one and only one given Schema. Notice that STAllele is an Allele (see Figure 1) defined in a MLST Schema, disjoint from Spa Allele and VNTAllele. So, we only associate to MLST typing information the concept of STAllele. This is necessary given the different ways in which the distinct alleles are defined in these typing methods.

The entity Spa Allele is used in the context of spa typing. Each spa typing information has a matching spa Allele, which corresponds to a specific sequence of repeats found as a result of the amplification of the locus of the spa gene of Staphylococcus aureus. In Figure 2 we can observe that a given Spa Allele has repeat profile parts, i.e., an entity that stores the index order of a given Spa Repeat in a given Spa Allele. Notice that each Spa Repeat may occur more than once in a given Spa Allele and that different Spa Alleles can have distinct number of repeats.

Notice also the difference between properties hasIdentifiedAllele and hasDefinedAllele relating respectively MLST and Locus concepts with the Allele concept. One could imagine that alleles associated to a given MLST instance could be obtained through the defined Schema, since property hasDefinedAllele allows to relate loci and alleles. But this is not the case. A Locus may have associated many alleles, with each of them belonging to many $M L S T$ instances, and hence we cannot identify the allele belonging to a particular MLST instance. That can be accomplished through property hasIdentifiedAllele which relates each MLST instance with its identified alleles. Although we did not add those kind of assertions in our ontology, we can still use this information to assert that the identified alleles for a given $M L S T$ instance are sufficient against a given Schema. We could even infer for which schemas a given $M L S T$ instance provides enough information.

It is also important to note that, by knowing only the Locus, it is possible to identify the Taxon that it belongs to, using the is OfTaxon property (see Figure 1).

Additional information for each class, such as sample collection date and other id, are described through data properties. For instance, the class Allele has data properties such as id and date entered. The class Isolate has data properties such as sample collection date and date entered.

Whenever possible, we reuse concepts from and establish relations with other ontologies as indicated in Table 1. An Agent is a concept imported from the FOAF ontology [25] and it can be a person, a group of persons or even an institution. In TypOn it allows the description of a person or a group of persons who have curated the 
information about the isolate, who have submitted that information to the database and who own the isolate. These relations are described by the object properties curated by, sent by and owned by, respectively. Reusing the Agent concept is extremely useful because it will allow, for instance, the use of TypOn together with the Web Access Control ontology [30] for defining access control levels in microbial typing databases, an important issue as mentioned above. Several applications in defining access control have been widely discussed and are well known to the research community $[31,32]$.

All TypOn concepts were derived from the Basic Formal Ontology (BFO) [20], the Ontology for Biomedical Investigations (OBI) [23] and the Sequence Ontology (SO) [21] to ensure upper-level interoperability with other ontologies. In order to avoid huge imports in TypOn we have used OntoFox [33] to query and import only relevant concepts in top level ontologies. These are then imported as, and are available at, https://bitbucket.org/phyloviz/typon/ raw/master/imported.owl.

TypOn was also submitted to the BioPortal (https:// bioportal.bioontology.org/), hosted by the National Center of Biomedical Ontologies (NCBO), being also available at http://bioportal.bioontology.org/ontologies/TYPON.

Concepts such as Schema and Typing Information are qualities (BFO_0000019), i.e., a categorical property. As discussed, we have classified an Isolate as an organism (OBI_0100026). More details in Figure 4.

Figure 5 depicts the Typon concepts that are related to the Sequence Ontology. Notice that we define Locus as a region (SO:0000001), since it is a named region on a genome.

Another example is the Spa Repeat concept which is a repeat unit (SO:0000657). Both Locus and Spa Repeat are also generically dependent continuant (BFO_0000031) since region (SO:0000001) and repeat unit (SO:0000657) concepts are subclasses of BFO_0000031 as defined in the sequence ontology (SO). Thus these concepts are related to both SO and BFO (see Figures 4 and 5).

\section{Use case}

In this section, we illustrate how we can represent typing information annotated with the TypOn ontology. Our example makes use of data regarding the characterization of a Staphylococcus aureus isolate for the purpose of this case. We will use the Turtle language [34] in the description of our isolate.

We can represent the isolate named Sa66296 as follows:

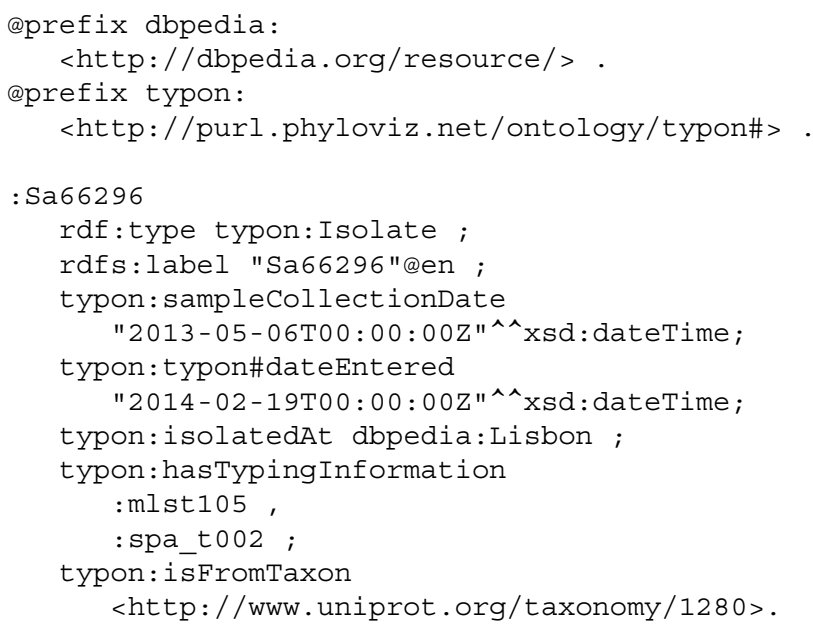

This is an instance of typon:Isolate labelled as "Sa66296". rdfs:label is an instance of rdf:Property that may be used to provide a human-readable version for the name of a resource. We further specify that it has two pieces of typing information mlst105 and

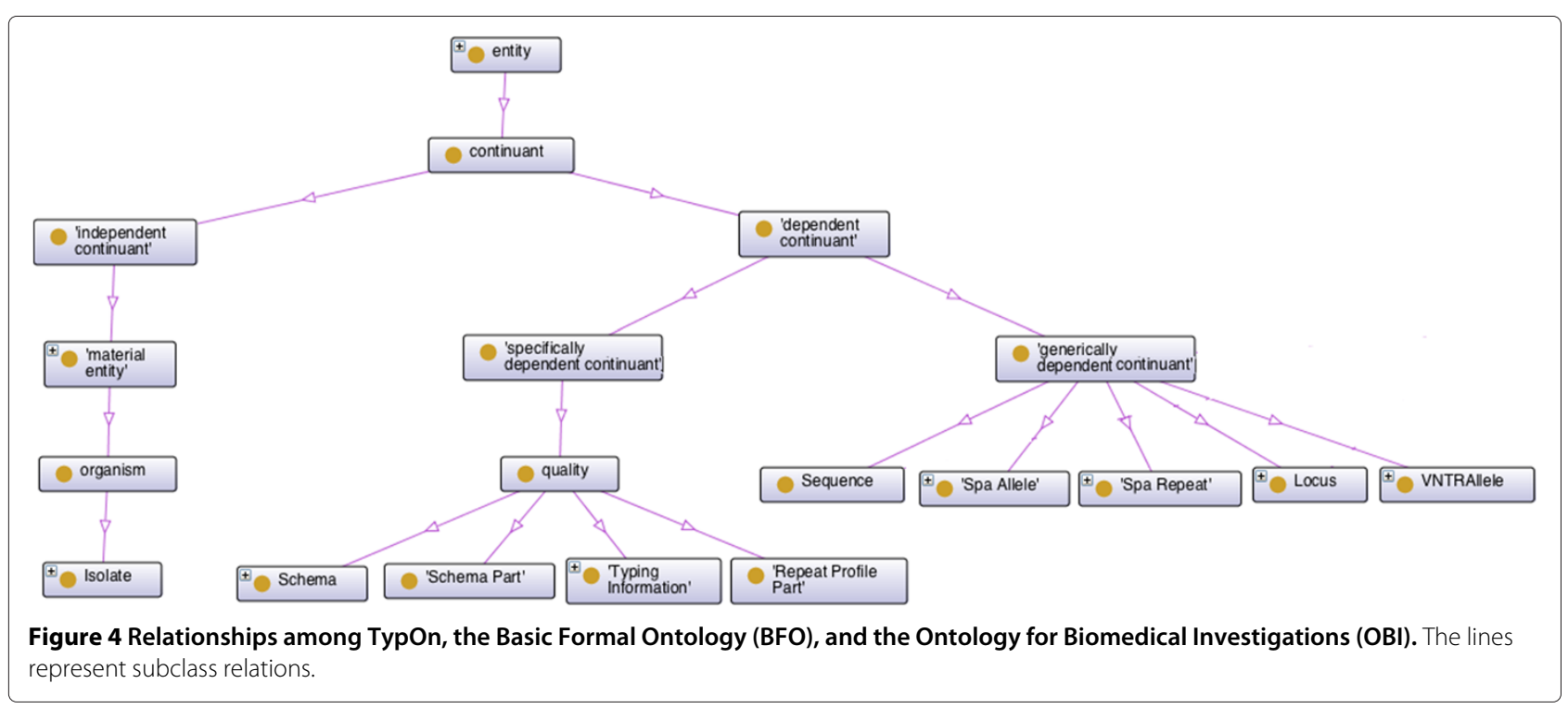




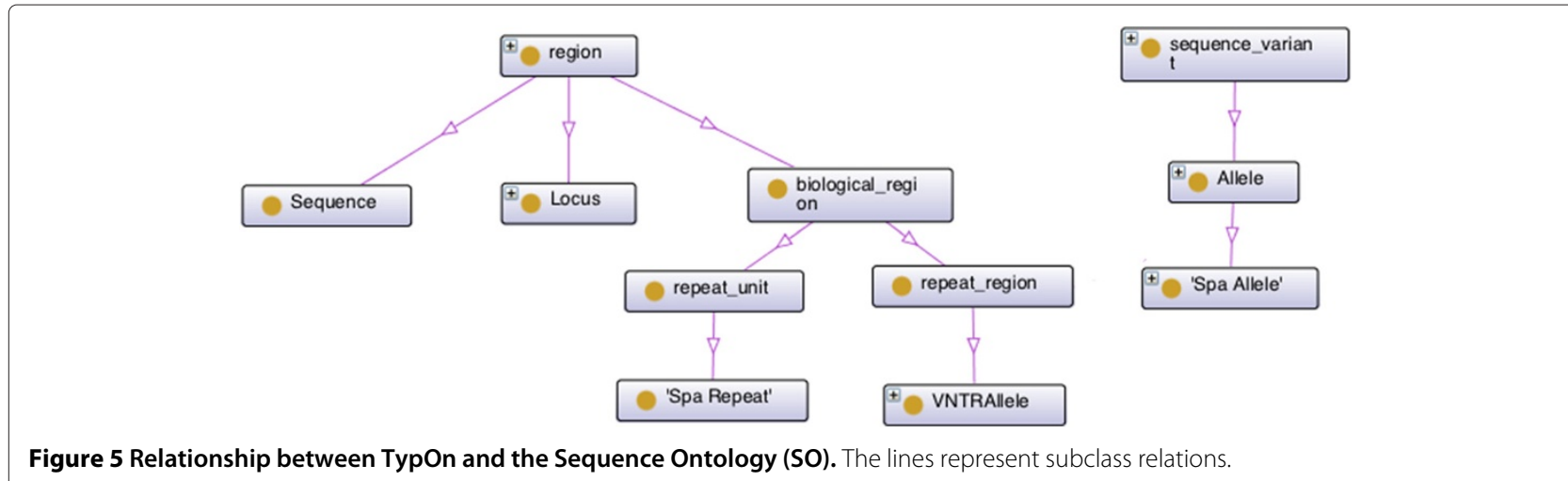

spa_t002 (instances of typon:MLST and typon:spaTyping, respectively). Thus, these pieces of typing information are other individuals annotated with our ontology. We can keep track of the dates when the isolate was collected and when the isolate was entered into the system using properties sampleCollectionDate and dateEntered, respectively. We also describe the origin of the isolate, with the individual labelled Lisbon which has type dbpedia-owl:Place. Note that this isolate was recovered in Lisbon, Portugal, represented as a resource in DBpedia. Figure 6 depicts the isolate information.

The individual labelled mlst 105 represents the sequence based typing method with a schema defined by the sequence of seven housekeeping loci, represented as follows:

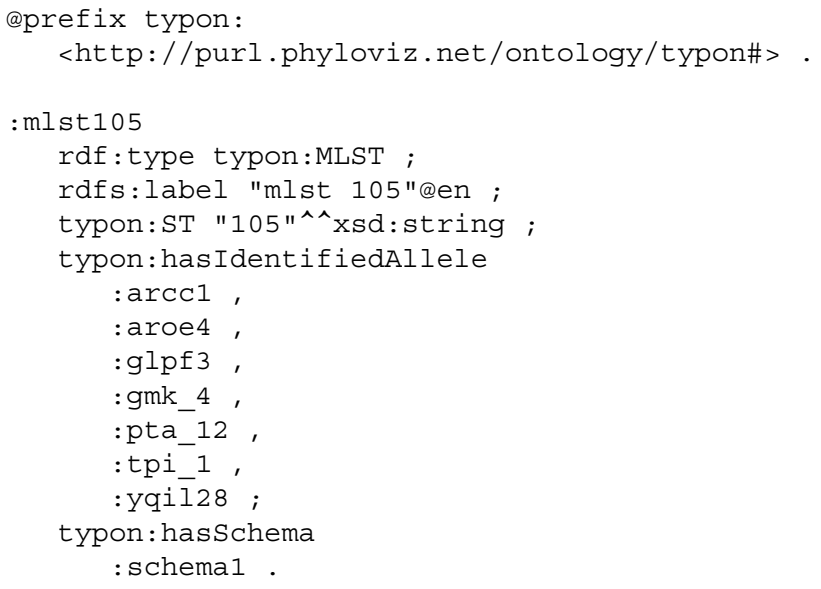

Note that the individual labelled schema1 identifies the seven housekeeping loci, using individuals of type typon:SchemaPart for keeping track of the index of each locus:

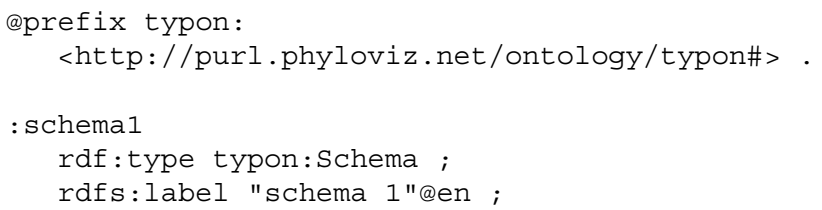

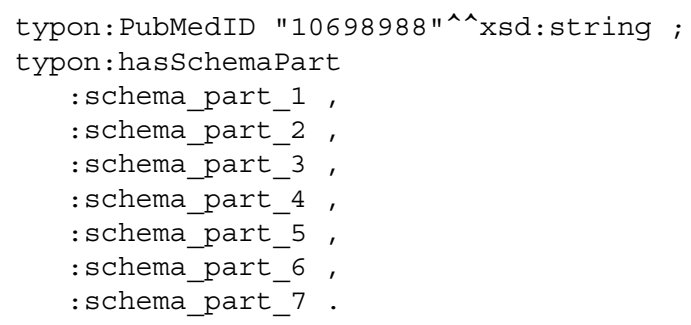

Let us now describe the individual that represent the arcc locus, the first locus in the considered schema as described by the individual labelled schema_part_1:

@prefix typon:

<http://purl.phyloviz.net/ontology/typon\#>.

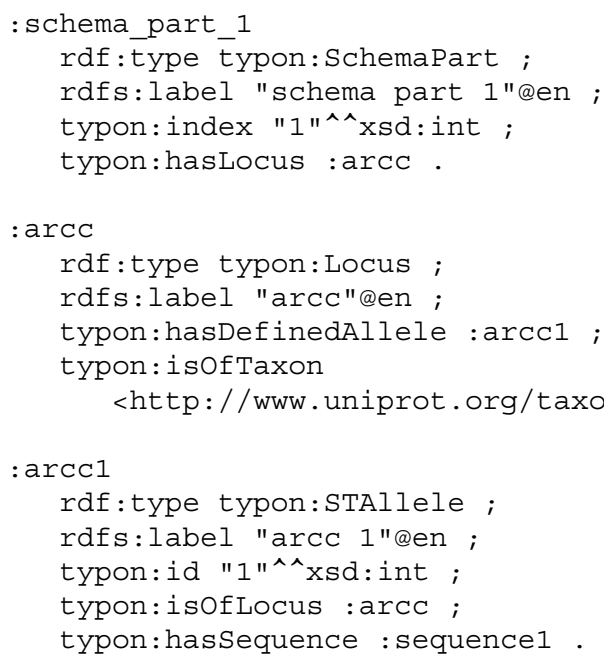

Note that the identified alleles of the loci can be directly obtained from the individual labelled mlst 105 . Furthermore all possible defined alleles can be obtained from the respective loci. It is also possible to obtain the locus that is associated to each allele, namely by property typon:isOfLocus. Figure 7 summarizes the representation of the MLST typing information concerning the isolate in our example. The complete example is available 


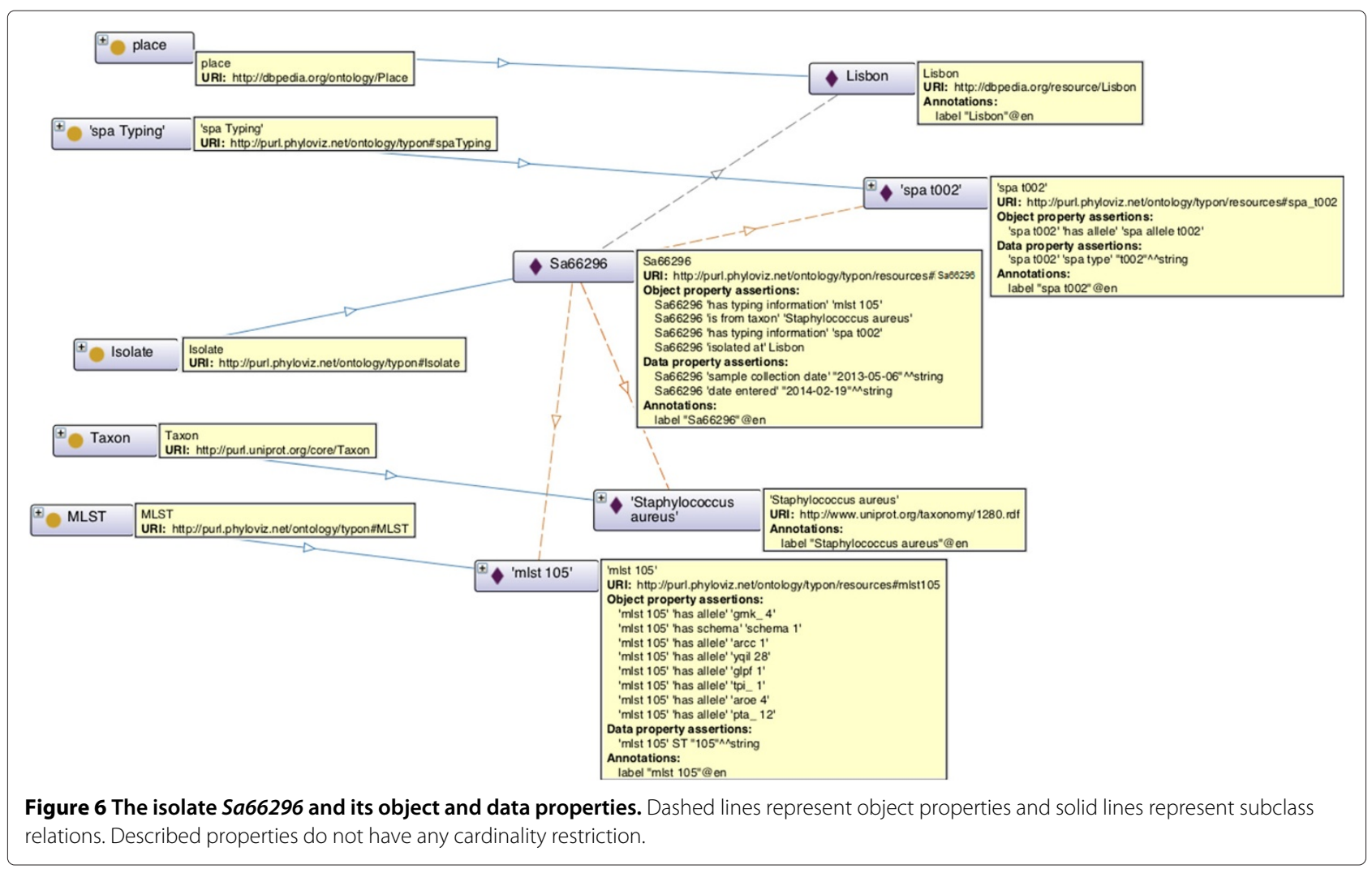

at https://bitbucket.org/phyloviz/typon/raw/master/test/ Sa66296.ttl.

\section{Annotating data}

In this section we discuss how to annotate a large dataset with the TypOn ontology and how to perform queries. We started by writing a D2RQ mapping for the data available for Neisseria spp, one of the databases available in our local BIGSdb [16] installation. D2RQ [35,36] is a mapping language and platform for treating nonRDF relational databases as virtual RDF graphs, aiming to expose RDBs on the semantic web. The mapping allows us to reuse existing vocabularies and ontologies, such as TypOn, to map relational schemas, such as the one underlying BIGSdb. We note that our mapping is not exhaustive and that we just annotated part of the data with TypOn ontology. The mapping is available at https://bitbucket.org/phyloviz/typon/raw/master/test/ BIGSdb_d2r_mapping.ttl.

Even though the D2RQ web app provides a SPARQL endpoint, it turns out that queries may take a long time to complete, causing high loads in the underlying database, and the web app may also become irresponsive. To overcome this issue we used the tool dump-rdf available with D2RQ to dump all triples and we uploaded them to a local instance of Virtuoso, which among other functionalities includes a highly efficient triple store (http://virtuoso. openlinksw.com/). Hence, a more responsive SPARQL endpoint is available at http://data.phyloviz.net/sparql, where we should select http://rest.phyloviz.net/neisseria/ as the default graph. All resources at http://rest.phyloviz. net/neisseria/ are also dereferenceable through rewrite rules against the SPARQL endpoint.

Let us consider a few SPARQL queries for illustration purposes. Imagine that we wanted to define a new MLST schema that includes loci $\operatorname{carB}, \operatorname{gln} A$, and rpiA. How can we find all isolates for which we already have typing information under this new schema? It turns out that we can answer this question with a SPARQL query:

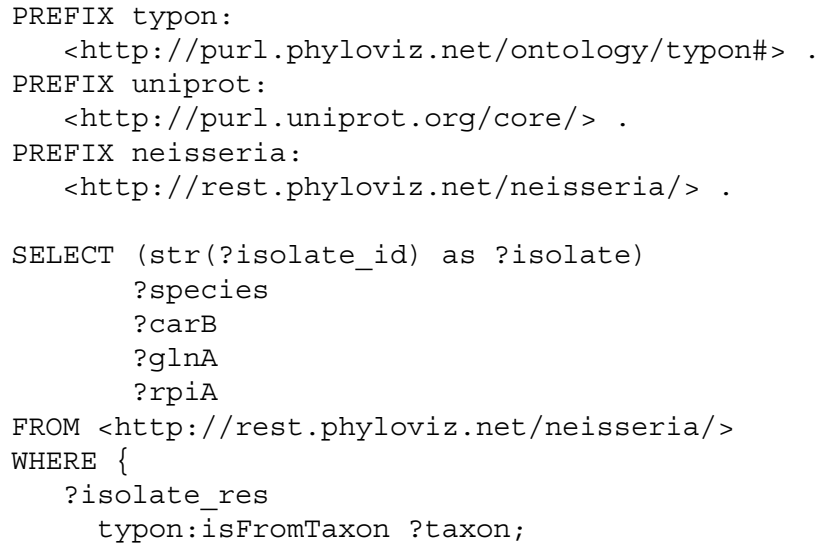




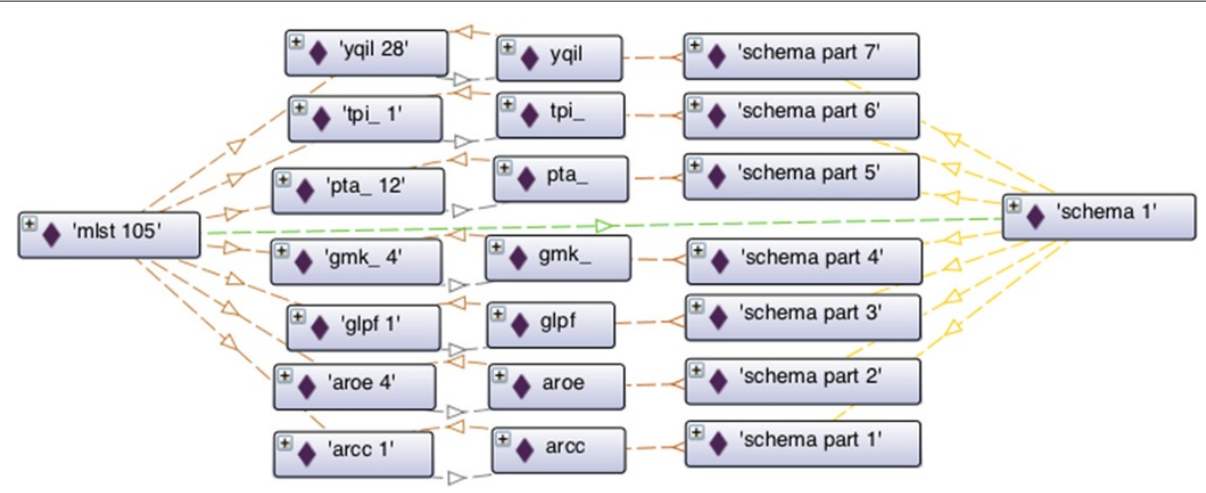

Figure 7 MLST typing information for isolate Sa66296. Dashed lines represent object properties. Instance m/st 105 of typon: MLST is related with instance schema 1 of typon: Schema through object property typon: hasSchema with cardinality of exactly one. Instance m/st 105 is also related with several instances of typon: Allele, e.g., instance aroe 4, through typon: hasldentifiedAllele. Instance schema 1 is related with several instances of typon: SchemaPart, e.g., instance schema part 2, through property typon: hasSchemaPart. Each instance of typon: SchemaPart is related with an instance of typon: Locus, e.g., instance schema part 2 is related with instance aroe, through property typon: hasLocus with cardinality of exactly one. Each instance of typon: Allele is related with an instance of typon: Locus, e.g., instance aroe 4 is related with instance aroe, through property typon: isOfLocus with cardinality of exactly one. Each instance of typon: Locus is also related with an instance of typon: Allele, e.g., instance aroe is related with instance aroe 4 through property typon: hasDefinedAllele.

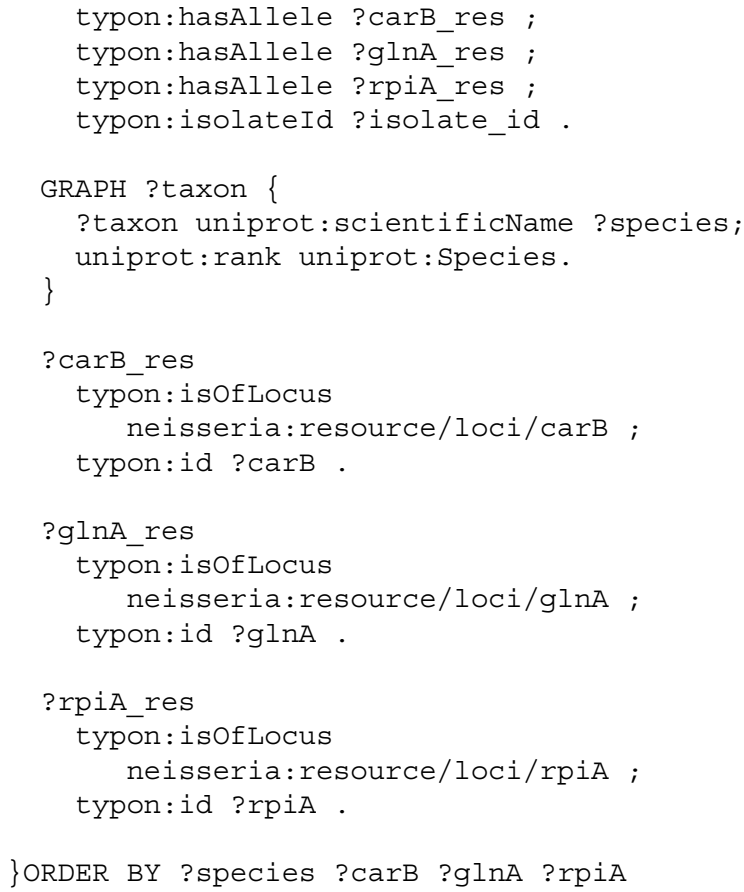

We can then submit this query to our endpoint at http:// data.phyloviz.net/sparql and our results include:

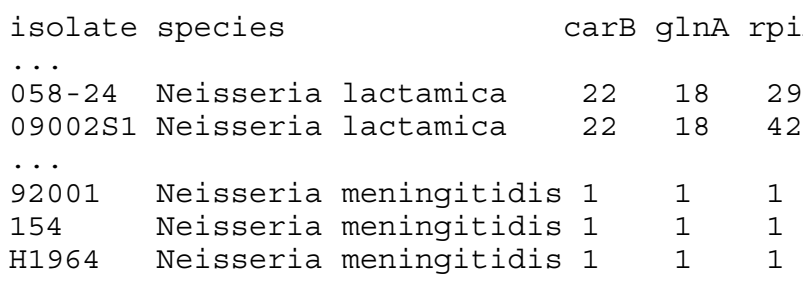

As another example, we may be interested in exploring the variability at the third locus in any MLST schema in our dataset, but only for isolates of Neisseria polysaccharea found in Canada. Taking into account the relationships defined in TypOn and Uniprot, we can retrieve this variability as follows through a federated query:

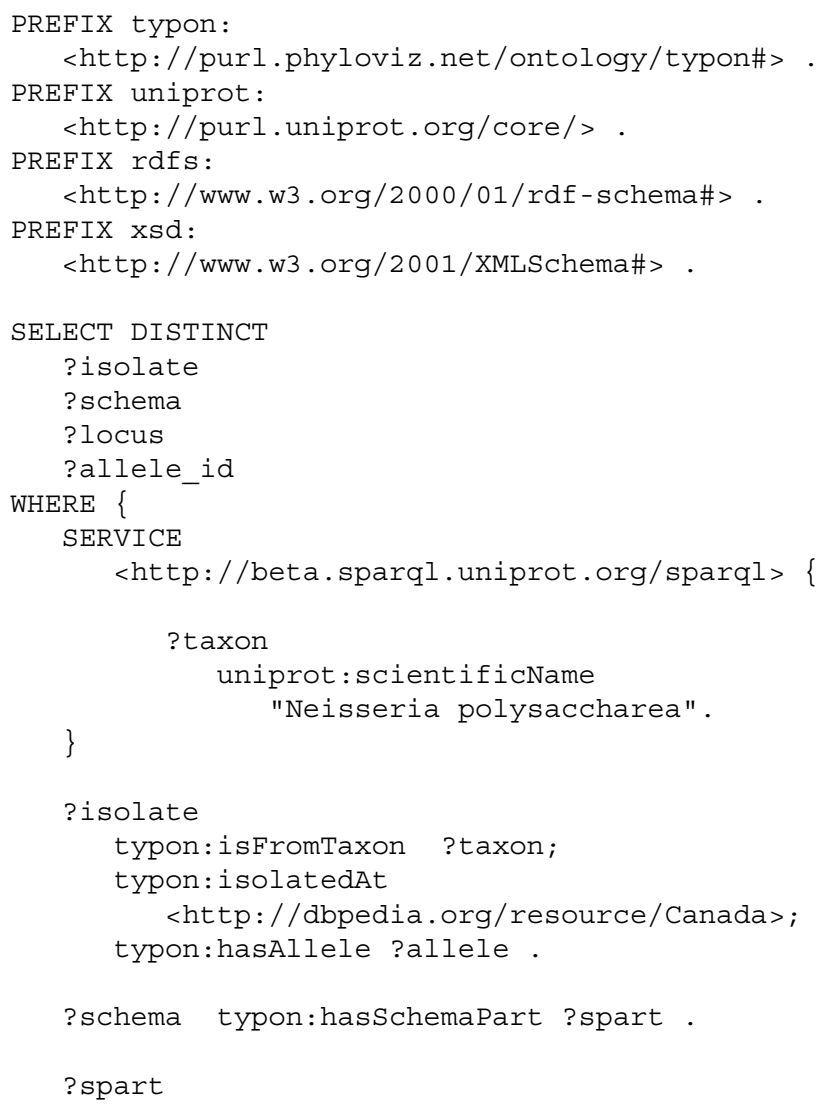




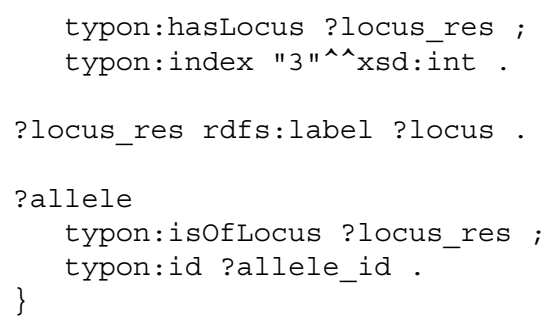

By submitting this query to http://data.phyloviz.net/ sparql, we obtain

$\begin{array}{llcc}\text { isolate } & \text { schema } & \text { locus allele_id } \\ \mathrm{db}: \text { isolates/ } & \mathrm{db}: \text { schemes/1 } & \text { aroE } & 286 \\ \quad 5194 & & & \\ \mathrm{db}: \text { isolates/ } & \mathrm{db}: \text { schemes/1 } & \text { aroE } & 289 \\ 5195 & & & \end{array}$

where the prefix $d b$ : stands for http://rest.phyloviz.net/ neisseria/resource/.

\section{Final remarks}

TypOn provides the basic concepts needed to establish the vocabulary and the semantic relationships for different sequence-based typing methods, and it is designed to allow further expansion. It was defined based on three different approaches to sequence-based typing: using the DNA sequence information directly, using the sequence of repeats in a DNA sequence, and for MLVA, using the number of repeats in a locus. Since these three approaches can be used to define many of the existing typing methods, TypOn can be easily expanded to encompass the newer multilocus typing techniques that are appearing based on NGS technologies, defined by expansion of the MLST concepts to larger numbers of genes [37] or by Single Nucleotide Polymorphism approaches, where each position on the genome can be viewed as a locus and the nucleotide present as an allele. Other advantages of this ontology is that it can provide a consistent link with legacy microbial typing techniques and provide a way to describe and annotate the evolution of specific typing schemas. This will be of paramount importance, if schemas that will be constructed by grouping loci from existing schemas or adding new loci, are to be designed and represented in an accurate way. This ontology is the first stepping stone on the implementation of a semantic web approach for the data repositories in this field. It lays the foundation for a common language that can be used to integrate and link data from different typing databases and for a complete merging of microbial typing with microbial genomics. Using the strategy discussed in the previous section (Annotating data), a SPARQL endpoint is already deployed for the Pubmlst MLST databases at http://pubmlst.org/sparql. This endpoint accesses data annotated using TypOn for MLST databases for 75 distinct bacterial species that are hosted at Pubmlst.org and further 29 species hosted externally to Pubmlst.org. A RESTful API is also being developed to facilitate data access without requiring the SPARQL endpoint. Future work will focus on expanding the ontology and creating and deploying RESTful APIs to perform not only custom querying but also automated submission and curation of data for authenticated users, in order to speed up and distribute the curating process, and ensure better quality and reproducibility of data in the field of microbial typing.

\section{Competing interests \\ The authors declare that they have no competing interests.}

\section{Authors' contributions}

All authors contributed to the development of the ontology. CV, APF, JAC, MR and MS created/edited textual definitions of ontology terms. CV, APF, MR and JAC have wrote and edited the manuscript. CV, MS and JAC have contributed with the individuals that exemplify the ontology. APF has annotated data in our local BIGSdb and JAC has helped him in the SPARQL queries examples. All authors discussed, read and approved both the ontology and the manuscript.

\section{Acknowledgements}

The work presented in this paper made use of data available at MLST.net [10], PubMLST [11] and Institut Pasteur MLST Databases [12]. This study was partly supported by the European Community grant FP7-278864-2

(PathoNgenTrace, http://www.patho-ngen-trace.eu/), and by national funds through FCT - Fundação para a Ciência e Tecnologia, under projects PTDC/EIA-CCO/118533/2010, EXCL/EEI-ESS/0257/2012, and

PEst-OE/EEI/LA0021/2013.

\section{Author details}

${ }^{1}$ INESC-ID, R. Alves Redol 9, 1000-029 Lisboa, Portugal. ${ }^{2}$ Instituto Superior de Engenharia de Lisboa, Instituto Politécnico de Lisboa, R. Cons. Emídio Navarro 1, 1959-007 Lisboa, Portugal. ${ }^{3}$ Instituto Superior Técnico, Universidade de Lisboa, Av. Rovisco Pais 1, 1049-001 Lisboa, Portugal. ${ }^{4}$ Instituto de Microbiologia, Instituto de Medicina Molecular, Faculdade de Medicina, Universidade de Lisboa, Av. Prof. Egas Moniz, 1649-028 Lisboa, Portugal. ${ }^{5}$ Department of Zoology, University of Oxford, Oxford, UK. ${ }^{6}$ Applied Maths NV, Keistraat 120, 98308 Sint-Martens-Latem, Belgium. ${ }^{7}$ Ridom GmbH, Mendelstr. 11, D-48149 Münster, Germany.

Received: 20 June 2014 Accepted: 6 October 2014

Published: 18 October 2014

\section{References}

1. Sá-Leão R, Pinto F, Aguiar S, Nunes S, Carriço JA, Frazão N, Gonçalves-Sousa N, Melo-Cristino J, de Lencastre H, Ramirez M: Analysis of invasiveness of pneumococcal serotypes and clones circulating in portugal before widespread use of conjugate vaccines reveals heterogeneous behavior of clones expressing the same serotype. J Clin Microbiol 2011, 49(1):1369-1375.

2. Struelens MJ: Consensus guidelines for appropriate use and evaluation of microbial epidemiologic typing systems. Clin Microbiol Infect 1996, 2(1):2-11.

3. Brueggemann AB, Griffiths DT, Meats E, Peto T, Crook DW, Spratt BG: Clonal relationships between invasive and carriage Streptococcus pneumoniae, and serotype- and clone-specific differences in invasive disease potential. J Infect Dis 2003, 187(9):1424-1432.

4. Allardet-Servent A, Bouziges N, Carles-Nurit MJ, Bourg G, Gouby A, Ramuz $M$ : Use of low-frequency-cleavage restriction endonucleases for DNA analysis in epidemiological investigations of nosocomial bacterial infections. J Clin Microbiol 1989, 27(9):2057-2061.

5. Carriço JA, Sabat AJ, Friedrich AW, Ramirez M, on behalf of the ESCMIDStudyGroupforEpidemiologicalMarkers(ESGEM): Bioinformatics in bacterial molecular epidemiology and public health: databases, tools and the next-generation sequencing revolution. Euro Surveill 2013, 18(4):20382. 
6. Almeida J, Tiple J, Ramirez M, Melo-Cristino J, Vaz C, Francisco AP, CarriçO $J A$ : An ontology and a rest api for sequence based microbial typing data. In JBI'2010. Lecture Notes in Computer Science, vol. 6620. Berlin Heidelberg: Springer; 2012:21-28.

7. Sabat AJ, Budimir A, Nashev D, Sa-Leao R, van Dijl Jm, Laurent F, Grundmann $\mathrm{H}$, Friedrich AW: Overview of molecular typing methods for outbreak detection and epidemiological surveillance. Euro Surveill 2013, 18(4):20380.

8. Spratt BG: Multilocus sequence typing: molecular typing of bacterial pathogens in an era of rapid DNA sequencing and the internet. Curr Opin Microbiol 1999, 2(3):312-316.

9. Maiden MC, Bygraves JA, Feil EJ, Morelli G, Russell JE, Urwin R, Zhang Q, Zhou J, Zurth K, Caugant DA, Feavers IM, Achtman M, Spratt BG Multilocus sequence typing: a portable approach to the identification of clones within populations of pathogenic microorganisms. Proc Natl Acad Sci U S A 1998, 95(6):3140-3145.

10. MLST: Multi Locus Sequence Typing. [http://www.mlst.net], Imperial College of London.

11. PubMLST. [http://pubmlst.org/], University of Oxford (UK).

12. Institut Pasteur MLST Databases. [http://www.pasteur.fr/mlst/], Pasteur Institute.

13. Harmsen D, Claus H, Witte W, Rothgänger J, Claus H, Turnwald D, Vogel U: Typing of methicillin-resistant Staphylococcus aureus in a university hospital setting by using novel software for spa repeat determination and database management. J Clin Microbiol 2003 41(12):5442-5448

14. Boucher HW, Corey GR: Epidemiology of methicillin-resistant Staphylococcus aureus. Clin Infect Dis 2008, 46(Suppl 5):344-349.

15. Lindstedt $B-A$ : Multiple-locus variable number tandem repeats analysis for genetic fingerprinting of pathogenic bacteria. Electrophoresis 2005, 26(13):2567-2582.

16. Jolley KA, Maiden MCJ: BIGSdb: Scalable analysis of bacterial genome variation at the population level. BMC Bioinformatics 2010, 11:595.

17. Jolley KA, Bliss CM, Bennett JS, Bratcher HB, Brehony C, Colles FM, Wimalarathna H, Harrison OB, Sheppard SK, Cody AJ, Maiden MCJ: Ribosomal multilocus sequence typing: universal characterization of bacteria from domain to strain. Microbiology 2012, 158(Pt 4):1005-1015.

18. Croucher NJ, Harris SR, Fraser C, Quail MA, Burton J, van der Linden M, McGee L, von Gottberg A, Song J-H, Ko KS, Pichon B, Baker S, Parry CM, Lambertsen LM, Shahinas D, Pillai DR, Mitchell TJ, Dougan G, Tomasz A, Klugman KP, Parkhill J, Hanage WP, Bentley SD: Rapid pneumococcal evolution in response to clinical interventions. Science 2011 331(6016):430-434

19. Harris SR, Feil EJ, Holden MT G, Quail MA, Nickerson EK, Chantratita N, Gardete S, Tavares A, Day N, Lindsay JA, Edgeworth JD, de Lencastre H, Parkhill J, Peacock SJ, Bentley SD: Evolution of MRSA during hospital transmission and intercontinental spread. Science 2010, 327(5964):469-474

20. Bittner $T$, Smith B: Normalizing medical ontologies using basic formal ontology. In Kooperative Versorgung, Vernetzte Forschung, Ubiquitäre Information (Proceedings of GMDS Innsbruck, 26-30 September 2004). Niebüll: Videel OHG; 2004:199-201.

21. Eilbeck K, Lewis SE, Mungall CJ, Yandell M, Stein L, Durbin R, Ashburner M: The sequence ontology: a tool for the unification of genome annotations. Genome Biol 2005, 6(5):44

22. Buttigieg PL, Morrison N, Smith B, Mungall CJ, Lewis SE, the ENVO Consortium: The environment ontology: contextualising biological and biomedical entities. J Biomed Semant 2013, 4:43.

23. Peters $B$, the $\mathrm{OBI}$ Consortium: Ontology for biomedical investigations. In International Conference on Biomedical Ontology (ICBO 2009). 2009. Available from Nature Precedings [http://dx.doi.org/10.1038/npre.2009. 3623.1]

24. Uniprot Core Ontology. [http://purl.uniprot.org/core/]

25. The friend of a friend(foaf) project. [http://www.foaf-project.org/]

26. The DBpedia Ontology. [http://wiki.dbpedia.org/Ontology]

27. Redaschi N, UniProt Consortium: UniProt in RDF: Tackling data integration and distributed annotation with the semantic web. In 3rd International Biocuration Conference. 2009. Available from Nature Precedings [http://dx.doi.org/10.1038/npre.2009.3193.1]
28. Brinkman RR, Courtot M, Derom D, Fostel JM, He Y, Lord P, Malone J, Parkinson H, Peters B, Rocca-Serra P, Ruttenberg A, Sansone S-A, Soldatova LN, Stoeckert CJ, Turner JA, Zheng J, the OBI Consortium: Modeling biomedical experimental processes with OBI. J Biomed Semant 2010, 1(Suppl 1):7.

29. Bizer C, Lehmann J, Kobilarov G, Auer S, Becker C, Cyganiak R, Hellmann S: DBpedia - a crystallization point for the web of data. Web Semant Sci Serv Agents World Wide Web 2009, 7(3):154-165.

30. Web Access Control. [http://www.w3.org/wiki/WebAccessControl]

31. Dumbill E: Support online communities with foaf: How the friend-ofa-friend vocabulary addresses issues of accountability and privacy. 2002. IBM's XML Watch, [http://web.archive.org/web/20070226182529/ http://www-128.ibm.com/developerworks/xml/library/x-foaf2.html]

32. Dumbill E: Tracking provenance of rdf data. 2003. IBM's XML Watch, [http://web.archive.org/web/20050428083208/http://www-106.ibm. com/developerworks/xml/library/x-rdfprov.html]

33. Xiang Z, Courtot $M$, Brinkman RR, Ruttenberg $A$, He Y: Ontofox: web-based support for ontology reuse. BMC Res notes 2010, 3(1):175

34. Beckett D, Berners-Lee T, Prud'hommeaux E, Carothers G: Turtle: Terse RDF Triple Language. 2012. [http://www.w3.org/TR/2012/WD-turtle20120710/]

35. Bizer C, Seaborne A: D2RQ-treating non-RDF databases as virtual RDF graphs. In Proceedings of the 3rd International Semantic Web Conference (ISWC2004). Hiroshima: Springer; 2004.

36. Bizer C, Cyganiak R, Garbers G, Maresch O, Becker C: The D2RQ Platform v0. 7-Treating Non-RDF Relational Databases as Virtual RDF Graphs. 2009. User Manual and Language Specification [http://wifo5-03. informatik.uni-mannheim.de/bizer/d2rq/spec/20090810/]

37. Maiden MCJ, van Rensburg MJJ, Bray JE, Earle SG, Ford SA, Jolley KA, McCarthy ND: MLST revisited: the gene-by-gene approach to bacterial genomics. Nat Rev Microbio/ 2013, 11(10):728-736.

doi:10.1186/2041-1480-5-43

Cite this article as: Vaz et al.: TypOn: the microbial typing ontology. Journal of Biomedical Semantics 2014 5:43.

\section{Submit your next manuscript to BioMed Central and take full advantage of:}

- Convenient online submission

- Thorough peer review

- No space constraints or color figure charges

- Immediate publication on acceptance

- Inclusion in PubMed, CAS, Scopus and Google Scholar

- Research which is freely available for redistribution 